\title{
A Multiscale Change Detection Technique Robust to Registration Noise
}

\author{
Lorenzo Bruzzone, Francesca Bovolo, and Silvia Marchesi \\ Department of Information and Communication Technologies \\ Via Sommarive, 14 \\ I-38050 Trento, Italy \\ lorenzo.bruzzone@ing.unitn.it
}

\begin{abstract}
This paper addresses the problem of unsupervised change detection in multitemporal very high geometrical resolution remote sensing images. In particular, it presents a study on the effects and the properties of the registration noise on the change-detection process in the framework of the polar change vector analysis (CVA) technique. According to this study, a multiscale technique for reducing the impact of residual misregistration in unsupervised change detection is presented. This technique is based on a differential analysis of the direction distributions of spectral change vectors at different resolution levels. The differential analysis allows one to discriminate sectors associated with residual registration noise from sectors associated with true changes. The information extracted is used at full resolution for computing a change-detection map where geometrical details are preserved and the impact of residual registration noise is strongly reduced.
\end{abstract}

Keywords: Change detection, change vector analysis, registration noise, multitemporal images, very high geometrical resolution images, multiscale techniques, remote sensing.

\section{Introduction}

The availability of images acquired by satellite sensors on the same geographical area at different times makes it possible to identify and label possible changes occurred on the ground. In this context, the new generation of passive sensors (e.g., Ikonos, Quickbird, SPOT-5), which can acquire images with very high geometrical resolution, potentially can identify changes occurred on the ground with a high geometrical precision. However, the change-detection problem on VHR images is more complex than in the case of medium resolution images, and requires the development of proper advanced change-detection techniques.

In the literature, several unsupervised change-detection methods have been proposed for the analysis of medium resolution multispectral images [1]. Among them, a widely used unsupervised technique is the change vector analysis (CVA). Although CVA was used in several different application domains, only recently a rigorous and theoretically well defined framework for polar CVA has been introduced in the literature [2]. This framework is based on the analysis of the distribution of 
spectral change vectors (SCVs) in the polar domain and makes it possible to better analyze the properties of the change-detection problem. A basic assumption in the CVA technique is that multitemporal images acquired at different times are similar to each other, but for the presence of changes occurred on the ground. Unfortunately, this assumption is seldom completely satisfied due to differences in atmospheric and sunlight conditions, as well as in the acquisition geometry. In order to fulfill the similarity assumption, radiometric and geometric corrections, co-registration, and noise reduction are required. However, it is not possible to reach the ideal conditions with the pre-processing. This is particularly critical in the co-registration procedure, which usually results in a residual misalignment between images (registration noise) which affects the accuracy of the change-detection process leading to a sharp increase of the number of false alarms [2-5]. The effects of the registration noise (RN) are very critical in presence of multitemporal images acquired by VHR sensors. This is due to the complexity of the co-registration of VHR images, which mainly depends on the fact that last generation sensors acquire data with different view angles, thus resulting in multitemporal images with different geometrical distortions.

This paper analyzes the properties of the residual RN in multitemporal VHR images in the context of the polar framework for CVA. Then, according to this theoretical analysis, an adaptive multiscale technique is presented that aims at reducing the impact of the registration noise in the final change-detection map.

\section{Notation and Background}

Let us consider two multispectral images $\mathbf{X}_{\mathbf{1}}$ and $\mathbf{X}_{\mathbf{2}}$ acquired over the same geographical area at different times $t_{1}$ and $t_{2}$. Let us assume that these images do not show significant radiometric differences and that they are perfectly coregistered. Let $\Omega=\left\{\omega_{n}, \Omega_{c}\right\}$ be the set of classes of unchanged and changed pixels to be identified. In greater detail, $\omega_{n}$ represents the class of unchanged pixels, and $\Omega_{c}=\left\{\omega_{c_{1}}, \ldots, \omega_{c_{K}}\right\}$ the set of the $K$ possible classes (kinds) of changes occurred in the considered area. In order to analyze the effects of misregistration in change detection on VHR images and to define a proper technique for reducing the errors induced from registration noise on the change-detection map, we take advantage from the theoretical polar framework for unsupervised change detection based on the CVA proposed in [2]. As in [2], for simplicity, in the following the study is developed by considering a 2-dimensional feature space (however, the analysis can be generalized to the case of more spectral channels).

The CVA technique computes a multispectral difference image $\left(\mathbf{X}_{D}\right)$ by subtracting the feature vectors associated to each corresponding spatial position in the two considered images. Each SCV is usually implicitly represented in polar coordinates, with its magnitude $\rho$ and direction $\vartheta$ defined as:

$$
\vartheta=\tan ^{-1}\left(\frac{X_{1, D}}{X_{2, D}}\right) \quad \text { and } \quad \rho=\sqrt{\left(X_{1, D}\right)^{2}+\left(X_{2, D}\right)^{2}}
$$

where $X_{b, D}$ is the random variable representing the $b$-th component (spectral channel) of the multispectral difference image $\mathbf{X}_{D}$. 
Let us define the magnitude-direction $(M D)$ domain that includes all change vectors for the considered scene as a circle in the Polar representation:

$$
M D=\left\{\rho \in\left[0, \rho_{\max }\right] \text { and } \vartheta \in[0,2 \pi)\right\}
$$

where $\rho_{\max }=\max \left\{\sqrt{\left(X_{1, D}\right)^{2}+\left(X_{2, D}\right)^{2}}\right\}$. The complete modeling of a change-detection problem requires to identify regions associated with: i) unchanged and ii) changed pixels. Thus the $M D$ domain can be split into two parts: i) circle $C_{n}$ of no-changed pixels; and ii) annulus $A_{c}$ of changed pixels. This can be done according to the optimal Bayesian threshold value $T$ that separates pixels belonging to $\omega_{n}$ from pixel belonging to $\Omega_{c}$.

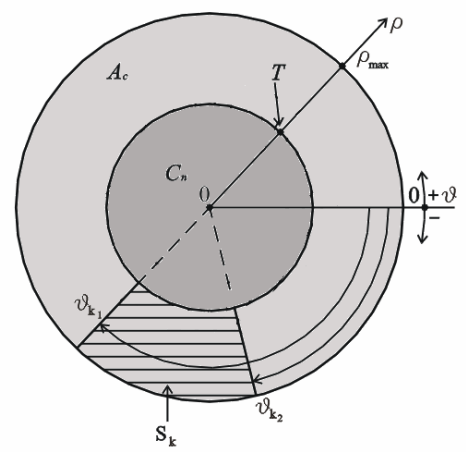

Fig. 1. Representation of the regions of interest for the CVA technique in the Polar coordinate system

Definition 1: the Circle of unchanged pixels $C_{n}$ is defined as $C_{n}=\{\rho, \vartheta: 0 \leq \rho<T$ and $0 \leq \vartheta<2 \pi\} . C_{n}$ can be represented in the polar domain as a circle with radius $T$.

Definition 2: the Annulus of changed pixels $A_{c}$ is defined as $A_{c}=\left\{\rho, \vartheta: T \leq \rho \leq \rho_{\max }\right.$ and $\left.0 \leq \vartheta<2 \pi\right\}$. $A_{c}$ can be represented in the Polar domain as a ring with inner radius $T$ and outer radius $\rho_{\max }$.

The previous definitions have been based on the values of the magnitude, independently on the direction variable. A further important definition is related to sectors in the Polar domain, which are mainly related to the direction of the SCVs and therefore to the kind of changes occurred on the ground.

Definition 3: the Annular sector $S_{k}$ of change $\omega_{k} \in \Omega_{\mathrm{c}}$ is defined as $S_{k}=\left\{\rho, \vartheta: \rho \geq T\right.$ and $\left.\vartheta_{k_{1}} \leq \vartheta<\vartheta_{k_{2}}, 0 \leq \vartheta_{k_{1}}<\vartheta_{k_{2}}<2 \pi\right\} . S_{k}$ can be represented in the Polar domain as a sector of change within $A_{c}$ and bounded from two angular thresholds $\vartheta_{k_{1}}$ and $\vartheta_{k_{2}}$.

The three regions are depicted in Fig. 1. For space constraint, we refer the reader to [2] for a complete set of definitions and for a discussion on them. 


\section{Properties of Registration Noise}

In this section we analyze the effects and the properties of the $\mathrm{RN}$ in the polar CVA domain according to the use of a VHR reference image. To this purpose, let us consider a Quickbird image acquired in July 2006 on the Trentino area (Italy) (see Fig. 3.b and section $\mathrm{V}$ for further details on this image). From this image, two simulated multitemporal datasets are generated made up of the original image $\left(\mathbf{X}_{1}\right)$ and of one shifted version of it $\left(\mathbf{X}_{\mathbf{2}}\right)$ (shifts of 2 and 6 pixels were considered). These two simulated data sets allow understanding the behavior of the $\mathrm{RN}$ components in a controlled experimental setup, as there are no true changes in the two images and there are no differences in their radiometric properties. Let us observe that the application of the CVA technique to $\mathbf{X}_{\mathbf{1}}$ and itself when images are perfectly co-registered leads to a multispectral difference image made up of SCVs with all zero components. Thus the representation in polar coordinates of SCVs collapses in a single point at the origin. An analysis of the histograms obtained after applying the CVA technique to the red and the near infrared spectral channels of image $\mathbf{X}_{1}$ and its 2 and 6 pixels shifted versions (Fig. 2.a and b) allows one to derive two main important observations on the properties of the RN:

1. The RN results in a cluster in the circle of unchanged pixels. The variance of the cluster increases by increasing the $\mathrm{RN}$. This effect is mainly related to the comparisons of non-perfectly aligned pixels that however correspond to the same object in the two images (see grey circles in Fig. 2).

2. The RN also results in significant clusters of pixels in the annulus of changed pixels. This is mainly due to the effects of the comparison of pixels which belong to different objects (pixels associated with detail and border regions) and is particularly significant in VHR images due to both the presence of regular structures and the presence of very high frequency regions. These clusters give raise to annular sectors having properties very similar to annular sectors of changed pixels (see black circles in Fig. 2), and are associated with specific misregistration components. We define these annular sectors as annular sectors of registration noise $\left(S_{R N_{i}}\right)$. They are very critical because they cannot be distinguished from sectors of true changes resulting in a significant false alarm rate in the change-detection process.

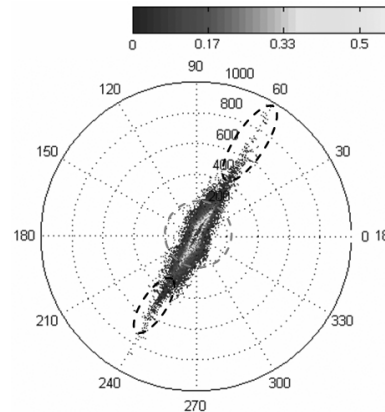

(a)

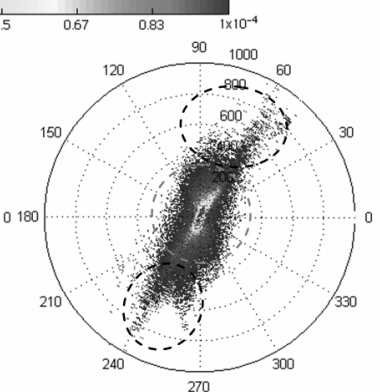

(b)

Fig. 2. Histograms in the Polar coordinate system obtained after applying CVA to the simulated data sets that show (a) 2 pixels of residual registration noise, and (b) 6 pixels of residual registration noise 


\section{Proposed Multiscale Technique for Registration Noise Reduction}

In order to identify the annular sectors of registration noise and to reduce their impact on the change-detection process, we propose a multiscale technique based on the analysis of the behaviors of SCVs in the polar domain at different resolution levels. One of the main assumptions at the basis of the proposed technique is that we expect that true significant changes are associated with objects with a non-negligible size and that misregistration appears in the difference image with relatively thin structures. Therefore, if we reduce the resolution of images, we implicitly decrease the impact of the registration noise with respect to that on the original scene. The lower is the geometrical resolution, the lower is the probability of identifying in the polar representation annular sectors of registration noise. This means that at low resolution levels in the annulus of changed pixels mainly sectors due to the presence of true changes on the ground can be detected. However, in order to obtain a change-detection map characterized by a good geometrical fidelity, we should work at full resolution. On the basis of this analysis, we propose a multiscale strategy for reducing the impact of the registration noise on the change-detection map, which is based on the following two steps: i) multiscale decomposition of the multitemporal images; and ii) differential analysis of the statistical behaviours of the direction distribution of SCVs in the annulus of changed pixels at different scales.

The first step aims at computing a multiscale representation of the information present in the considered images. Irrespectively on the multiscale decomposition approach considered, the multiresolution decomposition step produces two sets of images $\boldsymbol{X}_{M S_{i}}=\left\{\mathbf{X}_{i}^{0}, \ldots, \mathbf{X}_{i}^{n}, \ldots, \mathbf{X}_{i}^{N-1}\right\}$, where the subscript $i(i=1,2)$ denotes the acquisition date, and the superscript $n(n=0,1, \ldots, N-1)$ indicates the resolution level. Images in $\boldsymbol{X}_{M S_{i}}$ show different tradeoffs between registration noise and geometrical detail content.

In the second step, the set of multiscale difference images $\boldsymbol{X}_{D}=\left\{\mathbf{X}_{D}^{0}, \ldots, \mathbf{X}_{D}^{n}, \ldots, \mathbf{X}_{D}^{N-1}\right\}$ is computed by applying the CVA technique to each corresponding pair $\left(\mathbf{X}_{1}^{n}, \mathbf{X}_{2}^{n}\right)$ of images in $\boldsymbol{X}_{M S_{1}}$ and $\boldsymbol{X}_{M S_{2}}$. The distributions of the direction of SCVs at different resolution levels are then analyzed. In greater detail, the information extracted from the spectral difference image $\mathbf{X}_{D}^{N-1}$ at the lowest considered resolution level is exploited for reducing the effects of registration noise in the change-detection process performed at full resolution. Sectors associated with true changes on the ground are detected at lower scales and this information is exploited at full resolution for recovering change information, by properly processing sectors characterized by the presence of registration noise. It is expected that reducing the resolution the pixels associated with registration noise tend to disappear given their properties that usually result in small and thin structures. On the opposite, true changes (that usually have a non-negligible size) will be reduced in terms of size but not completely smoothed out. It follows that, as the resolution decreases, the marginal conditional density of the direction of pixels in $A_{c}$ (i.e., pixels candidate to be true changed pixels) $p(\vartheta \mid \rho \geq T)^{1}$ decreases in annular sectors of registration noise $\left(S_{R N_{i}}\right)$ whereas it remains nearly constant in annular sectors of true changes $\left(S_{k}\right)$.

\footnotetext{
${ }^{1} T$ is the threshold value that separates the circle of unchanged pixels from the annulus of changed pixels.
} 
According to the previous analysis, in order to discriminate annular sectors of registration noise $\left(S_{R N_{i}}\right)$ from annular sectors of true changes $\left(S_{k}\right)$ we define the marginal conditional density of registration noise $(R N)$ in the direction domain as:

$$
\hat{p}^{R N}(\vartheta \mid \rho \geq T)=C\left[p^{0}(\vartheta \mid \rho \geq T)-p^{N-1}(\vartheta \mid \rho \geq T)\right]
$$

where $p^{0}(\vartheta \mid \rho \geq T)$ and $p^{N-1}(\vartheta \mid \rho \geq T)$ are the marginal conditional densities of the direction of pixels in $A_{c}$ at the full resolution and at the lowest considered resolution level $N$-1, respectively, and $C$ is a constant defined such that $\int_{-\infty}^{+\infty} \hat{p}^{R N}(\vartheta \mid \rho \geq T)=1$. The proposed procedure associates the sectors where $\hat{p}^{R N}(\vartheta \mid \rho \geq T)$ exceeds a predefined threshold value $T_{R N}$ with sectors of registration noise $S_{R N_{i}}$, i.e.,

$$
S_{R N_{i}}=\left\{\rho, \vartheta: \rho \geq T, \vartheta_{i_{1}} \leq \vartheta \leq \vartheta_{i_{2}} \text { and } \hat{p}^{R N}(\vartheta \mid \rho \geq T) \geq T_{R N} \text { with } 0 \leq \vartheta_{i_{1}}<\vartheta_{i_{2}}<2 \pi\right\} \text {. }
$$

In this way we can apply the CVA technique to magnitude and direction variables in the polar domain and automatically reject patterns in the annulus of changed pixels associated with $S_{R N_{i}}$, thus reducing the false alarms due to the registration noise.

\section{Experimental Results}

In order to assess the reliability of the proposed analysis and the effectiveness of the proposed technique for reducing the impact of registration noise in the changedetection process, a multitemporal data set made of two images acquired by the Quikbird satellite on the Trentino area (Italy) in October 2005 and July 2006 was considered. In the pre-processing phase the two images were: i) pan-sharpened by applying the Gram-Schmidt procedure implemented in the ENVI software package [6] to the panchromatic channel and the four bands of the multispectral image; ii) radiometrically corrected; iii) co-registered. The registration process was carried out by using a polynomial function of order 2 according to 14 ground control points (GCPs), and applying a nearest neighbor interpolation. The final data set was made up of two pan-sharpened multitemporal and multispectral images of $984 \times 984$ pixels with a spatial resolution of $0.7 \mathrm{~m}$ that have a residual miregistration of about 1 pixel on GCPs. Fig. 3.a and b show the pan-sharpened near infrared channels of images $\mathbf{X}_{\mathbf{1}}$ and $\mathbf{X}_{2}$, respectively. Between the two acquisition dates two kinds of changes occurred: i) new houses were built on rural area ( $\omega_{c_{1}}$, white circles in Fig. 3.b); and ii) some roofs in the industrial and urban area were rebuilt $\left(\omega_{c_{2}}\right.$, black and light gray circles in Fig. 3.b). In order to allow a quantitative evaluation of the effectiveness of the proposed method, a reference map (which includes 21248 changed pixels and 947008 unchanged pixels) was defined according to the available prior knowledge on the considered area (see Fig. 3.c).

Fig. 4.a shows the histograms in polar coordinates obtained after applying the CVA technique to the red and the near infrared spectral channels, which better emphasize the changes occurred in the considered test area. From the analysis of these histograms and 


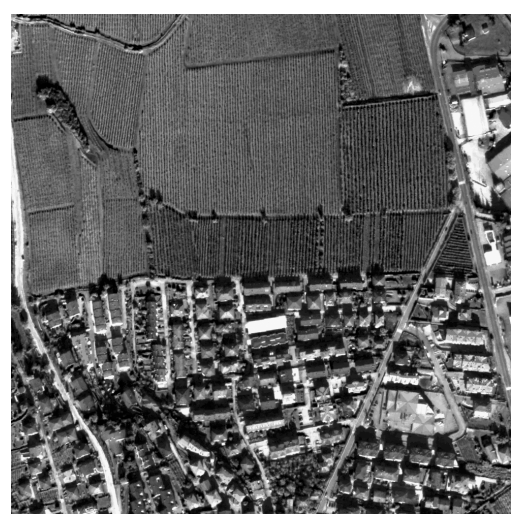

(a)

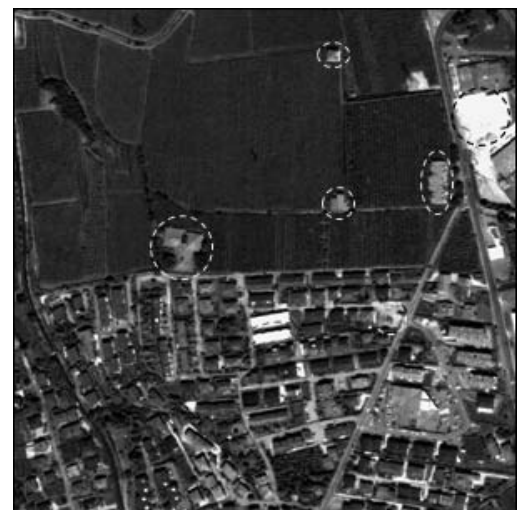

(b)

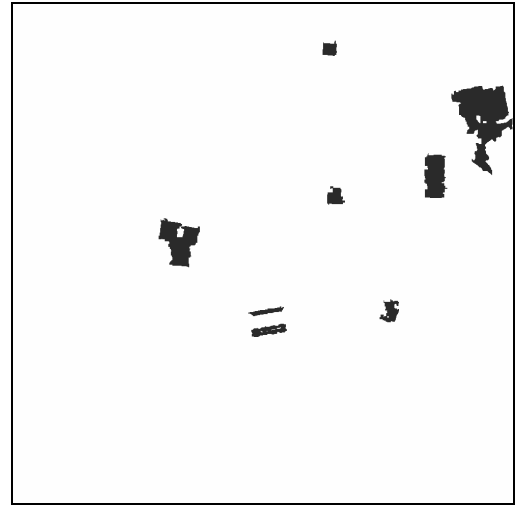

(c)

Fig. 3. Near infrared spectral channels of images of the Trentino area (Italy) acquired by the Quickbird VHR multispectral sensor in: (a) October 2005; and (b) July 2006 (changes occurred between the two acquisition dates appear in dashed white, black and gray circles). (c) Changedetection reference map.

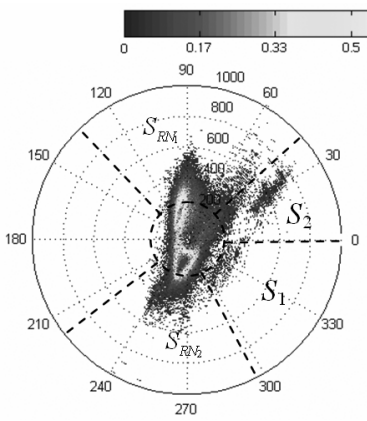

(a)

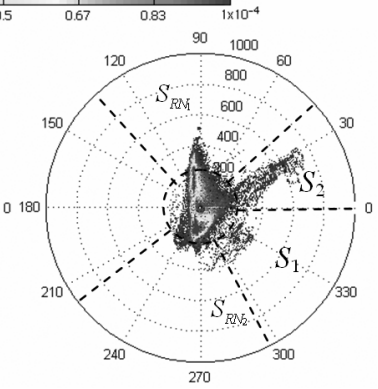

(b)

Fig. 4. Histograms in the Polar coordinate system of (a) $\mathbf{X}_{D}^{0}$ and (b) $\mathbf{X}_{D}^{4}$ 
of the available reference map, the annular sectors $S_{1}$ and $S_{2}$ associated with true changes $\omega_{c_{1}}$ and $\omega_{c_{2}}$, respectively, can be identified (see Fig. 4.a). As expected, the two clusters associated with $\omega_{c_{1}}$ and $\omega_{c_{2}}$ show a high magnitude and a preferred direction. All the other annular sectors: i) can be considered statistically meaningless due to the low occurrence of patterns; or ii) are associated with the residual registration noise $\left(S_{R N_{i}}\right)$.

As we are dealing with unsupervised change detection, all annular sectors including a significant amount of pixels are candidate to be associated with true changes on the ground, and can not be excluded a priori. In order to distinguish registration noise sectors from true change sectors, we computed two multiscale sets of images $\boldsymbol{X}_{M S_{1}}=\left\{\mathbf{X}_{1}^{0}, \ldots, \mathbf{X}_{1}^{4}\right\}$ and $\boldsymbol{X}_{M S_{2}}=\left\{\mathbf{X}_{2}^{0}, \ldots, \mathbf{X}_{2}^{4}\right\}$ applying the Daubechies -4 stationary wavelet transform [7] to images $\mathbf{X}_{1}$ and $\mathbf{X}_{2}$. The set of spectral difference images $\boldsymbol{X}_{D}=\left\{\mathbf{X}_{D}^{0}, \ldots, \mathbf{X}_{D}^{4}\right\}$ was then computed applying the CVA technique to $\boldsymbol{X}_{M S_{1}}$ and $\boldsymbol{X}_{M S_{2}}$.

According to the proposed technique a decision threshold $T$ that separates the annulus of unchanged pixels $\left(A_{c}\right)$ from the circle of no-changed pixels $\left(C_{n}\right)$ was computed ( $T$ was set to 220 , which was assumed to be valid for all resolution levels). A visual analysis of the statistical distribution of SCVs at resolution levels 0 and 4 (see Fig. 4) points out that in some annular sectors the density of pixels varies significantly, whereas in others it is nearly stable. This is confirmed by observing Fig. 5.a, which shows the behaviors of the marginal conditional densities of the direction in $A_{c}$ at resolution level $0\left[p^{0}(\vartheta \mid \rho \geq T)\right]$ and $4\left[p^{4}(\vartheta \mid \rho \geq T)\right]$. In order to identify annular sectors $S_{R N_{i}}$, the behavior of $\hat{p}^{R N}(\vartheta \mid \rho \geq T)$ was derived (see Fig. 5.b). Accordingly, the decision threshold $T_{R N}$ was set to $3 \times 10^{-3}$ and the 2 annular sectors having registration noise were identified, i.e., $S_{R N_{1}}=\left\{\rho, \vartheta: \rho \geq 220\right.$ and $\left.37^{\circ} \leq \vartheta<143^{\circ}\right\}$, and $S_{R N_{2}}=\left\{\rho, \vartheta: \rho \geq 220\right.$ and $\left.232^{\circ} \leq \vartheta<293^{\circ}\right\}$ (see Fig. 4).

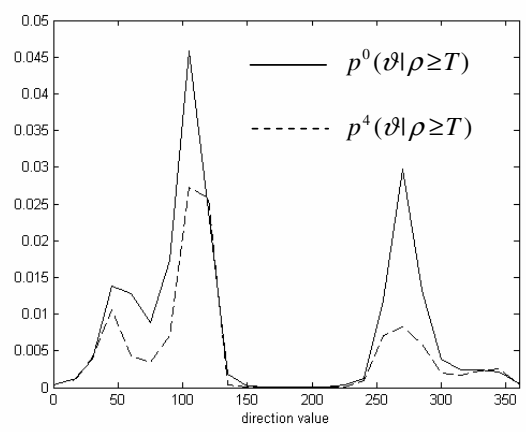

(a)

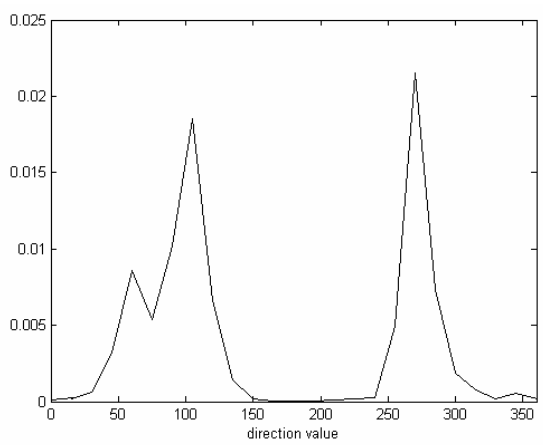

(b)

Fig. 5. Behaviors: (a) of the marginal conditional densities of the direction in $A_{c}$ at full resolution $p^{0}(\vartheta \mid \rho \geq T)$ (level 0$)$ and at the lowest resolution $p^{4}(\vartheta \mid \rho \geq T)$ (level 4) for the considered data set; and (b) of $\hat{p}^{D R N}(\vartheta \mid \rho \geq T)$ for the considered data set 
Table 1. Change-detection results obtained with the proposed multiscale technique and the standard technique that does not consider the presence of residual registration noise

\begin{tabular}{cccc}
\hline Technique & $\begin{array}{c}\text { False } \\
\text { alarms }\end{array}$ & $\begin{array}{c}\text { Missed } \\
\text { alarms }\end{array}$ & $\begin{array}{c}\text { Overall } \\
\text { error }\end{array}$ \\
\hline Proposed & 9138 & 3812 & 12950 \\
Standard & 55924 & 6354 & 62278 \\
\hline
\end{tabular}

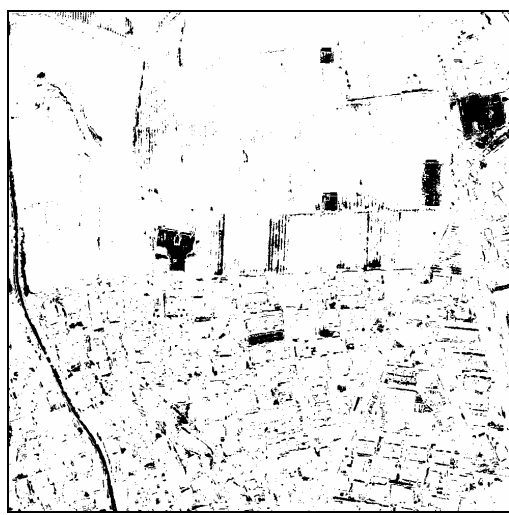

(a)

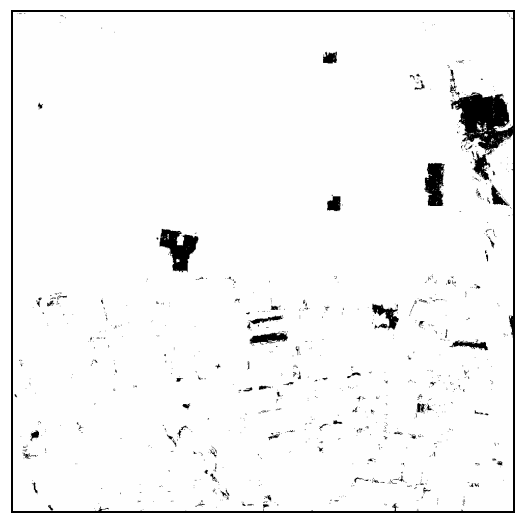

(b)

Fig. 6. Change-detection maps obtained with: (a) the standard approach that does not consider the presence of residual registration noise; (b) the proposed multiscale technique

The identified sectors were then used for computing the final change-detection map. SCVs that fall into $C_{n}, S_{R N_{2}}$ and $S_{R N_{1}}$ were classified as belonging to $\omega_{n}$, whereas the other statistically significant clusters in $A_{c}$ (i.e., associated with sectors $S_{1}$ and $S_{2}$ ) were classified as belonging to $\omega_{c_{1}}$ and $\omega_{c_{2}}$, respectively. The obtained change-detection map was compared with the one yielded ignoring the presence of registration noise, which was computed assigning SCVs that fall into $C_{n}$ to $\omega_{n}$, and all statistically significant clusters in $A_{c}$ to the class of changes. Numerical results reported in Tab. 1 point out the effectiveness of the proposed technique, which sharply reduces both the overall error and the false alarm rate. These results are confirmed by the changedetection maps reported in Fig. 6. It is worth noticing that the obtained change-detection map is computed at full resolution level, and thus exhibits a high geometrical detail content.

\section{Conclusion}

This paper has addressed the problem of unsupervised change detection in VHR multitemporal remote sensing images in the context of a polar framework for CVA. In particular, a study on the properties of registration noise on VHR images has been presented, and an adaptive multiscale technique for reducing the effects of residual 
registration noise in unsupervised change detection has been proposed. The proposed technique exploits both the magnitude and the direction information of SCVs in the polar domain at different resolution levels. Annular sectors of registration noise are identified in an automatic way according to a differential analysis of the marginal distributions of the direction in the annulus of changed pixels at different scales. The pixels included in these sectors are then rejected in the change-detection process at full resolution level. Experimental results, obtained on a pair of VHR multitemporal images acquired by the Quikbird sensor on a mixed urban and rural areas, confirmed the effectiveness of the proposed technique in reducing the effects of the registration noise.

\section{References}

1. Singh, A.: Digital change detection techniques using remotely-sensed data. Int. J. Rem. Sens. 10(6), 989-1003 (1989)

2. Bovolo, F., Bruzzone, L.: A Theoretical Framework for Unsupervised Change Detection Based on Change Vector Analysis in Polar Domain. IEEE Transactions on Geoscience and Remote Rensing 45(1), 218-236 (2007)

3. Dai, X., Khorram, S.: The effects of image misregistration on the accuracy of remotely sensed change detection. IEEE Transactions on Geoscience and Remote Sensing 36, 15661577 (1998)

4. Townshend, J.R.G., Justice, C.O., Gurney, C.: The impact of misregistration on change detection. IEEE Transactions on Geoscience and Remote Sensing 30, 1054-1060 (1992)

5. Bruzzone, L., Cossu, R.: An adaptive approach for reducing registration noise effects in unsupervised change detection. IEEE Transactions on Geoscience and Remote Sensing 41(11), 2455-2465 (2003)

6. ENVI User Manual. Boulder, CO: RSI (2003), http://www.RSInc.com/envi

7. Bovolo, F., Bruzzone, L.: A detail-preserving scale-driven approach to change detection in multitemporal SAR images. IEEE Transactions on Geoscience and Remote Sensing 43(12), 2963-2972 (2005) 